\title{
Melting of Binary Mixtures of Nylon 6 and Nylon 66. A Study by Pressure-Volume-Temperature Measurements
}

\author{
Kenji Matsumura, Takehiko Shimomura, Tsunetoshi Matsuda, ${ }^{*}$ \\ and Matsuo Hirami ${ }^{*, \dagger}$ \\ Unitika Research Laboratories, Inc., 23 Kozakura, Uji, Kyoto 611-0021, Japan \\ * Research and Development Center, Unitika Ltd., \\ 23 Kozakura, Uji, Kyoto 611-0021, Japan
}

(Received January 28, 1999)

\begin{abstract}
Pressure-volume-temperature $(P V T)$ measurements on binary mixtures of nylon 6 and nylon 66 were performed for melt-spun monofilament samples. During heating in the isobaric modes (form 20 to $180 \mathrm{MPa}$ ), two definite volume changes corresponding to the melting of nylon 6 and nylon 66 in mixtures were observed in the composition range where nylon 6 is the major part. From observed volume change at the melting, the heat of fusion $\Delta H_{\mathrm{m}}$ was estimated using the Clausius-Clapeyron equation. Thus, the amounts of coexisting two crystalline phases of both nylon components at a given composition can be estimated. A series of estimated $\Delta H_{\mathrm{m}}$ is self-consistent and reasonable. However, $\Delta H_{\mathrm{m}}$, obtained from the estimated values at finite pressures by extrapolating as $P \rightarrow 0$, are 33 and $28\left(\mathrm{cal} \mathrm{g}^{-1}\right)$ for nylon 6 and nylon 66 , and smaller than the literature data of $45\left(\mathrm{calg}^{-1}\right)$. The cause for this difference is discussed.

KEY WORDS Binary Mixtures / Clausius-Clapeyron Equation / Degree of Crystallinity / Finite Size Crystal / Heat of Fusion / Melting / Nylon 6 / Nylon 66 / Polymer Blend / Pressure-Volume-Temperature /
\end{abstract}

Investigation using pressure-volume-temperature $(P V T)$ measurements has been done over broad areas of polymer science and engineering, probably due to the fact that the volume-temperature coefficient at constant pressure: $\alpha$ and the volume-pressure coefficient at constant temperature: $\beta$ can be obtained from the measurement. From a practical viewpoint, information obtained from $P V T$ measurements are essential for application to polymer processing, and from the more academic viewpoint, data of $\alpha$ and $\beta$ are needed for the analysis of entropy change on the phase transition of melting, as made early for ordinary synthetic polymers by many researchers ${ }^{1-4}$ and recently for dimer liquid crystals by Abe and et al. ${ }^{5}$

Apart from these main-stream subjects, our PVT study is confined to melting of binary mixture systems of two crystalline polymers where both components are missible and crystallize separately. We believe that the required conditions above are satisfied in binary mixtures of nylon 6 and nylon 66, we have undertaken the present work, on the melting of nylon 6/nylon 66 mixtures. The experimental results are discussed from various aspects, especially, attention is directed to examine the applicability of the Clausius--Clapeyron equation to such binary systems.

\section{EXPERIMENTAL}

Pellets of nylon $6\left(M_{w}=16000\right)$ and nylon 66 $\left(M_{w}=22000\right)$ were blended in an extruder at various ratios of both nylons, namely, nylon $6 /$ nylon $66: 100 / 0$, $80 / 20,70 / 30,60 / 40,50 / 50,(40 / 60),(30 / 70)$ and $0 / 100$, and melt-spun blend samples of monofilament with diameter of $c a .1 .0 \mathrm{~mm}$ were obtained. The blend samples of $(40 / 60)$ and $(30 / 70)$ were used only in a preliminary study of the measurement.

\footnotetext{
${ }^{\dagger}$ To whom all correspondence should be addressed.
}

As PVT apparatus, Shimadzu model PVT200 tester was used. The apparatus consists of a cell containing several grams of sample and Wood's metal as confining fluid. Each melt-spun monofilament sample was cut in pieces (each with length $c a .5 \mathrm{~mm}$ ), and $c a .1 \mathrm{~g}$ of these pieces collected and $10 \mathrm{~g}\left(\mathrm{ca} .1 \mathrm{~cm}^{3}\right)$ of Wood's metal $\left(\mathrm{mp} 58^{\circ} \mathrm{C}\right)$ were confined in the cell. Measurements were made to determine changes in specific volume as a function of temperature under a constant pressure ranging from 20 to $180 \mathrm{MPa}$ at a fixed interval (40 MPa). As the heating rate, $1^{\circ} \mathrm{C} \mathrm{min}^{-1}$ was employed.

\section{RESULTS AND DISCUSSION}

As a preliminary study, we performed PVT measurements for two mixture samples of nylon 6 (N6) and nylon 66 (N66), (N6/N66):(40/60) and (30/70), and in volume-temperature diagram for both mixture samples, we could not find any definite change in volume at the melting temperature of nylon 6 . This shows that in the composition range where the content of nylon 6 is small (less than $50 \%$ ), the existence of nylon 6-crystalline phase is not appreciable in contrast to the predominance of nylon 66-crystalline phase. Thus, in this work, we used nylon 6/nylon 66 mixtures in the composition range where the content of nylon 6 is $50 \%$ or more, in addition to the pure nylon 6 and nylon 66 as control.

Figure 1 shows volume-temperature diagram in the isobaric modes $(P=20,60,100,140,180 \mathrm{MPa})$ for nylon 6 / nylon 66 mixtures, namely (N6/N66) : (100/0), $(80 / 20)$, (70/30), (60/40), (50/50) and $(0 / 100)$. We examined the experimental data shown in Figure 1, using the thermodynamic relationship of phase transition. In the classical thermodynamics, the Clausius-Clapeyron equation for the solid-liquid phase transition is,

$$
T_{\mathrm{m}} \frac{\mathrm{d} P}{\mathrm{~d} T_{\mathrm{m}}}=\frac{\Delta H_{\mathrm{m}}}{\Delta V_{\mathrm{m}}}
$$



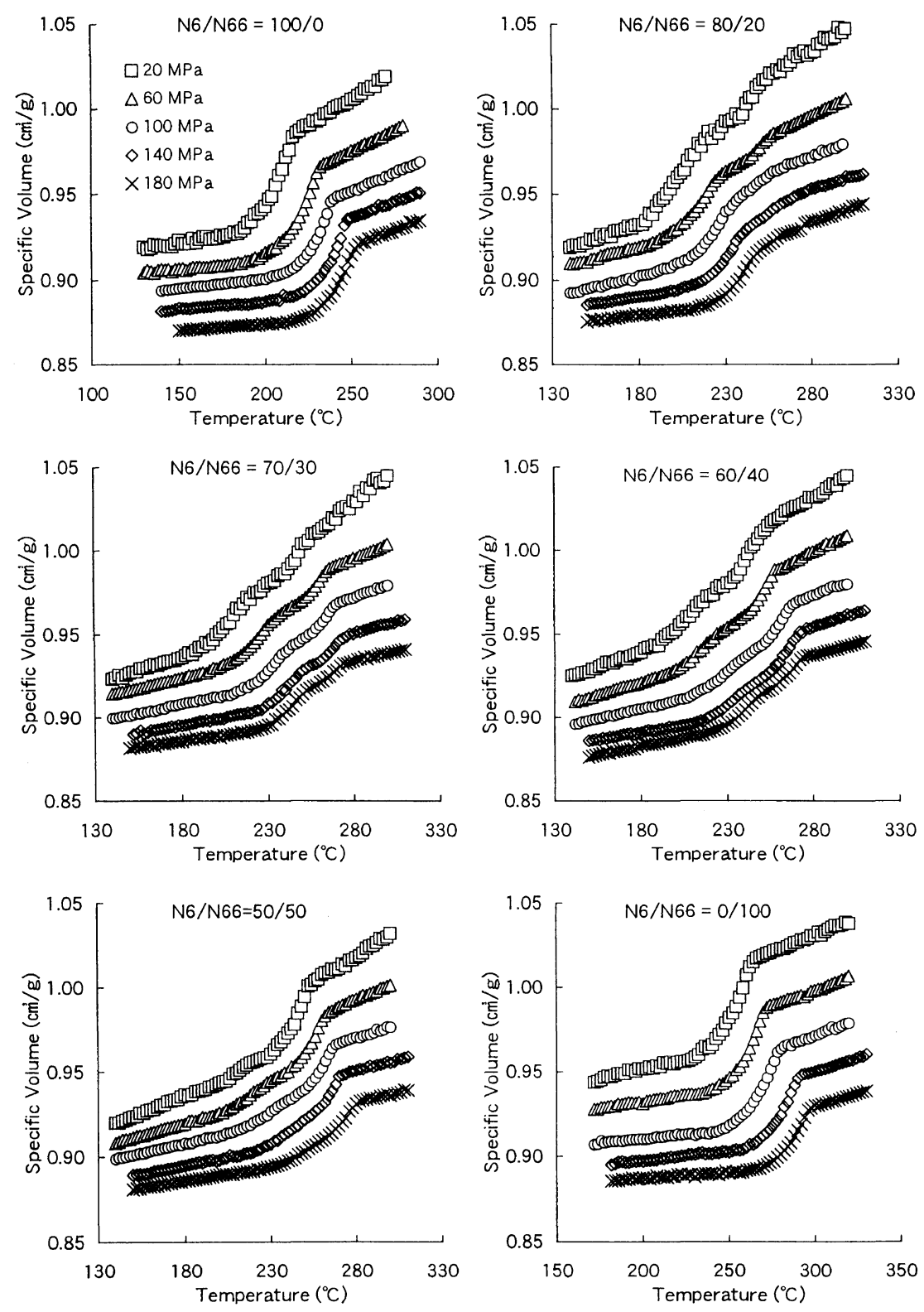

Figure 1. Volume vs. temperature in the isobaric modes $(P=20-180 \mathrm{MPa})$ for nylon $6(\mathrm{~N} 6)$, nylon 66 (N66) and mixtures : N6/N66 $=($ a) $100 / 0$, (b) $80 / 20$, (c) $70 / 30$, (d) $60 / 40$, (e) $50 / 50$, and (f) $0 / 100$.

where $T_{\mathrm{m}}$ is the melting temperature $(K)$, and $\Delta V_{\mathrm{m}}$ and $\Delta H_{\mathrm{m}}$ are changes of volume and enthalpy accompanying melting of the crystalline phase. In eq 1, it is assumed implicitly that the solid phase is wholly-crystalline such as metals and organic substances of low molecular weight. When the relationship is applied to semicrystalline polymer systems, $\Delta V_{\mathrm{m}}$ and $\Delta H_{\mathrm{m}}$ should be replaced by $\Delta V_{\mathrm{m}}^{\prime}$ and $\Delta H_{\mathrm{m}}^{\prime}$, respectively, which are defined by,

$$
\Delta V_{\mathrm{m}}^{\prime}=\Delta V_{\mathrm{m}} / Y \quad \text { and } \quad \Delta H_{\mathrm{m}}^{\prime}=\Delta H_{\mathrm{m}} / Y
$$

where $(Y \times 100)$ is the degree of crystallinity \%. After substitution of eq 2 into eq 1 , in the right-hand side of eq $1, Y$ in numerator and in denominator cancel each other, and the relationship is reduced to the same expression of eq 1 . Then, for a semicrystalline polymer system, experimental data can be analyzed using eq 1 . There still remains a question concerning the applicability of eq 1 to the present binary system. The following analysis assumes that eq 1 is applicable to each component of both nylons in mixtures, and total enthalpy

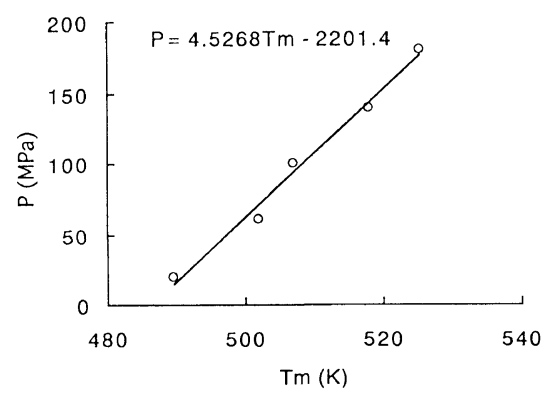

Figure 2. Plots of pressure $v s$. melting temperature of nylon 6 for the mixture of $(\mathrm{N} 6 / \mathrm{N} 66)=(70 / 30)$.

of melting of the mixture can thus be obtained as the sum of contributions from each component.

At given $T_{\mathrm{m}}$, by substituting the observed values of $\left(\mathrm{d} P / \mathrm{d} T_{\mathrm{m}}\right)$ and $\Delta V_{\mathrm{m}}$ into eq $1, \Delta H_{\mathrm{m}}$ was estimated. In Figure 2, the plot of $P$ vs. $T_{\mathrm{m}}$ of nylon 6 in the mixture of $(\mathrm{N} 6 / \mathrm{N} 66):(70 / 30)$ is shown as an example and the good linearity of the plot can be seen. The evaluation 
Table I. $\Delta H_{m}$ for nylon 6 , nylon 66 , and their mixtures

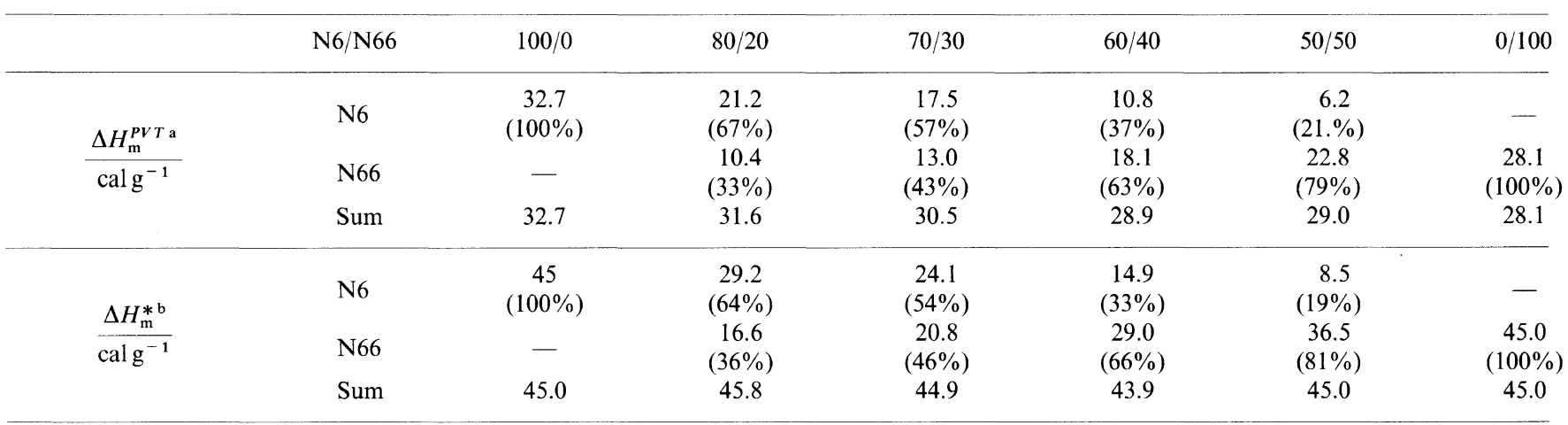

${ }^{\text {a }}$ Estimated from experimental data of $P V T$ measurements. ${ }^{\mathrm{b}}$ Converted to adjust $\Delta H_{\mathrm{m}}^{P V T}$ of N6 and N66 to literature data.

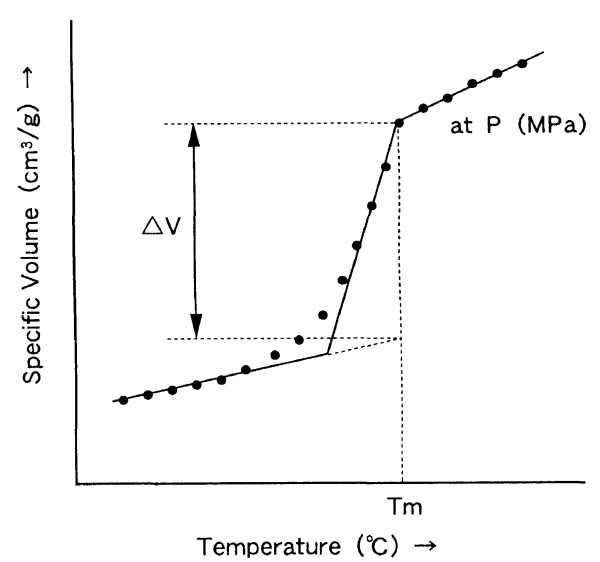

Figure 3. Evaluation procedure of $T_{\mathrm{m}}$ and $\Delta V_{\mathrm{m}}$ from volumetemperature curve in the isobaric mode.

procedure of $T_{\mathrm{m}}$ and $\Delta V_{\mathrm{m}}$ from the volume-temperature curve in diagram is illustrated in Figure $3 . \Delta H_{\mathrm{m}}$ obtained from the estimated values at finite pressures by extrapolating as $P \rightarrow 0$ are shown in Table I. For convenience, $\Delta H_{\mathrm{m}}$ estimated from $P V T$ measurements and $\Delta H_{\mathrm{m}}$ of literature data are designated as $\Delta H_{\mathrm{m}}^{P V T}$ and $\Delta H_{\mathrm{m}}^{*}$, respectively, and quantities related to nylon 6 and nylon 66 indicated by the suffix 1 and 2 . As can be seen in Table I, enthalpy changes accompanying the melting of crystalline phases of the pure nylon 6 and nylon 66 are $\Delta H_{\mathrm{m} 1}^{P V T}=33\left(\mathrm{calg}^{-1}\right)$ and $\Delta H_{\mathrm{m} 2}^{P V T}=28\left(\mathrm{cal} \mathrm{g}^{-1}\right)$, which are smaller than the literature data ${ }^{6} \Delta H_{\mathrm{m} 1}^{*}=\Delta H_{\mathrm{m} 2}^{*}=45$ $\left(\mathrm{cal} \mathrm{g}^{-1}\right)$. We introduce a parameter $f$, i.e., the conversion coefficient which adjusts the value of $\Delta H_{\mathrm{m}}^{P V T}$ to the literature data $\Delta H_{\mathrm{m}}^{*}$ by the relation,

$$
\Delta H_{\mathrm{m}}^{P V T}=f \Delta H_{\mathrm{m}}^{*}
$$

Apparently in this case, we have $f_{1}=0.73$ and $f_{2}=0.62$. The adjustment was similarly applied to coexisting two crystalline phases of both nylons in mixtures. Letting $W_{1}$ represent the fraction of nylon 6 , the values of $W_{1} \Delta H_{\mathrm{m} 1}^{P V T} / f_{1},\left(1-W_{1}\right) \Delta H_{\mathrm{m} 2}^{P V T} / f_{2}$ and their sum, i.e.,

$$
\Delta H_{\mathrm{m}}^{*}=W_{1} \Delta H_{\mathrm{m} 1}^{P V T} / \mathrm{f}_{1}+\left(1-W_{1}\right) \Delta H_{\mathrm{m} 2}^{P V T} / f_{2}
$$

are included in Table I. For all samples, converted $\Delta H_{\mathrm{m}}^{P V T}$, that is $\Delta H_{\mathrm{m}}^{*}$, represented by eq 4 lie in the same level, ca. $45\left(\mathrm{cal} \mathrm{g}^{-1}\right)$. This means that a series of estimated $\Delta H_{\mathrm{m}}$ is self-consistent and reasonable. This supports the validity of the assumption that eq 1 is applicable to each component of both nylons in mixtures as well as pure nylon 6 and nylon 66. The amounts of coexiting two crystalline phases of nylon 6 and nylon 66 at a given composition can thus be obtained. This is advantageous for application of $P V T$ measurements to binary mixture systems where both components crystallize separately.

Finally, the physical meaning of the conversion coefficient $f$ is discussed. The definition was made so that $f=1$ corresponds to the crystalline state of nylon 6 and nylon 66 which gives the heat of fusion $\Delta H_{\mathrm{m}}^{*}=$ $45\left(\mathrm{cal} \mathrm{g}^{-1}\right)$ cited in reference 6 , while $f=0$ refers to disappearance of the crystalline phase as the standard state. Literature data ${ }^{6}$ of $\Delta H_{\mathrm{m}}^{*}=45\left(\mathrm{calg}^{-1}\right)$ were estimated from both calorimetric measurments ${ }^{7-9}$ and melting temperature depression by monomeric diluents. ${ }^{10-12}$ In the former method, the heat of fusion of the wholly-crystalline state is estimated in a limiting case as the degree of crystallinity $\rightarrow 100 \%$. In the latter method, the melting temperature is determined by the final diappearance of crystallinity which corresponds to the highest ordered portion of the sample and longest crystallite should distribution of crystalline sequence exists. Although data of $\Delta H_{\mathrm{m}}^{*}$ reported by many researchers $^{7-12}$ scatter in the range of $40-45\left(\mathrm{cal} \mathrm{g}^{-1}\right)$, here $\Delta H_{\mathrm{m}}^{*}=45\left(\mathrm{cal} \mathrm{g}^{-1}\right)$ as cited in reference 6 was used.

$\Delta H_{\mathrm{m}}^{P V T}$ expresses the enthalpy change accompanying melting of the crystalline phase as a whole, which includes the surface of a finite size crystal and imperfection in bulk such as defects and less-ordered portions. Further, nylon molecules synthesized by condensation polymerization have an approximately most probable distribution of chain length, and some short molecular chains yields small size crystals which contribute to the lowering the enthalpy of fusion. The cause for the difference between $\Delta H_{\mathrm{m}}^{*}$ and $\Delta H_{\mathrm{m}}^{P V T}$ is attributed to factors mentioned above.

Summarizing the results, the conversion coefficient $f$ may be regarded as a parameter which represents the degree of perfection of crystalline phase. The estimate of $\Delta H_{\mathrm{m}}^{P V T}$ can be made independent of the degree of crystallinity. The usefulness of estimated $\Delta H_{\mathrm{m}}^{P V T}$ along with the relation of eq 4 as shown in this work is applicable to other semicrystalline polymer systems, for pure polymers and polymer mixtures. 


\section{CONCLUSIONS}

PVT measurements on binary mixtures of nylon 6 and nylon 66 for melt-spun monofilament samples were performed and the following conclusions were obtained.

(1) From volume-temperature diagram made in the isobaric modes (from 20 to $180 \mathrm{MPa}$ ) for nylon 6 , nylon 66 and mixtures (content of nylon 6 is $50-80 \%$ ), definite volume changes corresponding to the melting of nylon 6 and nylon 66 were observed.

(2) The Clausius-Clapeyron equation was applied to the two nylons in mixtures as well as pure nylon 6 and nylon 66, and from the observed volume change the enthalpy change $\Delta H_{\mathrm{m}}^{P V T}$ accompanying melting of crystalline phase was estimated. Amounts of coexisting two crystalline phases of both nylon components at a given composition in their mixtures were determined.

(3) $\Delta H_{\mathrm{m}}^{P V T}$ obtained from the estimated values at finite pressures by extrapolating as $P \rightarrow 0$, are 33 and 28 $\left(\mathrm{cal} \mathrm{g}^{-1}\right)$ for nylon 6 and nylon 66 which are smaller than the literature data of $\Delta H_{\mathrm{m}}^{*}=45\left(\mathrm{cal} \mathrm{g}^{-1}\right)$ estimated from calorimetric measurements and melting temperature depression by diluents. The factor $f=\left(\Delta H_{\mathrm{m}}^{P V T}\right)$ $\left.\Delta H_{\mathrm{m}}^{*}\right)$ may be regarded as a parameter representing the degree of perfection of crystalline phase.
Acknowledgment. This work was supported by the international joint research grant of NEDO (New Energy and Industrial Technology Development Organization). The authors wish to acknowledge with many thanks the advice and encouragement which they received from Prof. A. Abe.

\section{REFERENCES}

1. F. A. Quinn, Jr. and L. Mandelkern, J. Am. Chem. Soc., 80, 3178 (1958).

2. H. W. Starkweather, Jr. and R. H. Boyd, J. Phy. Chem., 64, 410 (1960).

3. R. P. Smith, J. Polym. Sci., A-2, 4, 869 (1966).

4. A. E. Tonelli, J. Chem. Phys., 52, 4749 (1970).

5. A. Abe and S. Y. Nam, Macromolecules, 28, 90 (1995); A. Abe, H. Furuya, R. N. Shimizu and S. Y. Nam, ibid., 28, 96 (1995).

6. L. Mandelkern, "Crystallization of Polymers," McGraw-Hill, New York, N.Y., 1963, Chapter 5-2.

7. R.C. Wilhoit and M. Dole, J. Phys. Chem., 57, 14 (1953).

8. M. Dole and B. Wunderlich, Makromol. Chem., 34, 29 (1959).

9. M. Inoue, J. Polym. Sci. A, 1, 2697 (1963).

10. H. Yamakawa, in "Kobunshi-no-Bussei III," M. Nakajima, Ed., Kyoritsu Shuppan, Tokyo, 1958, p 86.

11. G. B. Gechele and L. Crescentini, J. Appl. Polym. Sci., 7, 1349 (1963).

12. M. Hirami, Ph. D. thesis, Kyoto University (1971). 